\title{
Candida tropicalis distribution and drug resistance is correlated with ERG11 and UPC2 expression
}

\author{
Dan Wang ${ }^{1 \dagger}$, Na An ${ }^{2+}$, Yuwei Yang ${ }^{2}$, Xianggui Yang ${ }^{1}$, Yingzi Fan ${ }^{1}$ and Jiafu Feng ${ }^{2^{*}}$ (D)
}

\begin{abstract}
Background: Candida tropicalis (C. tropicalis) is an important opportunistic pathogenic Candida species that can cause nosocomial infection. In this study, we analyzed the distribution and drug susceptibility of $C$. tropicalis and the relationship between ERG11 and UPC2 expression and resistance to azole antifungal agents.
\end{abstract}

Methods: C. tropicalis was cultured and identified by Sabouraud Agar Medium, CHROM Agar Candida and ATB tests (Bio-Mérieux, France). Total RNA was extracted from the collected strains, and the ERG11 and UPC2 mRNA expression levels were analyzed by quantitative real-time PCR.

Results: In total, 2872 clinical isolates of Candida, including 319 strains of C. tropicalis, were analyzed herein; they were mainly obtained from the Departments of Respiratory Medicine and ICU. The strains were predominantly isolated from airway secretion samples, and the detection trend in four years was mainly related to the type of department and specimens. The resistance rates of $C$. tropicalis to fluconazole, itraconazole and voriconazole had been increasing year by year. The mRNA expression levels of ERG11 and UPC2 in the fluconazole-resistant group were significantly higher than they were in the susceptible group. In addition, there was a significant positive linear correlation between these two genes in the fluconazole-resistant group.

Conclusions: Overexpression of the ERG11 and UPC2 genes in C. tropicalis could increase resistance to azole antifungal drugs. The routine testing for ERG11 and UPC2 in high-risk patients in key departments would provide a theoretical basis for the rational application of azole antifungal drugs.

Keywords: Candida tropicalis, Drug resistance, ERG11, UPC2

\section{Introduction}

Candida tropicalis (C. tropicalis) is widely distributed in nature and is a common colonizer of the human skin, oral cavity and digestive tract [1]. Moreover, C. tropicalis is also an important opportunistic pathogenic Candida species that can cause nosocomial infection, and its isolation rate is second to that of Candida albicans

\footnotetext{
*Correspondence: jiafufengacad@foxmail.com; jiafufeng@aliyun.com

${ }^{\dagger}$ Dan Wang and Na An contributed equally to this work.

2 Department of Laboratory Medicine, Mianyang Central Hospital, Mianyang 621000, Sichuan Province, China

Full list of author information is available at the end of the article
}

(C. albicans) [2]. Patients with high-risk factors infected with $C$. tropicalis might suffer from infections in the lung [3], urogenital tract and intra-abdomen, as well as systemic infection [4-6]. Importantly, the increasing drugresistance of $C$. tropicalis has been reported in recent years. The global SENTRY antifungal surveillance report in 2013 showed that the resistance rate of C. tropicalis to fluconazole was $11.60 \%$ (in 31 countries total) [7]. Moreover, data from the China Invasive Fungal Resistance Monitoring Network (CHIF-NET) had shown that the resistance rate of $C$. tropicalis to fluconazole increased from $11.20 \%$ in 2009 to $42.70 \%$ in 2014 [8]. Therefore, the risk factors involved in C. tropicalis infection, the 
genotyping of drug-resistant strains, and the mechanism of drug resistance have caused widespread concern in recent years [9].

The resistance of $C$. tropicalis to azole antifungal drugs is mainly due to mutations and/or the overexpression of ergosterol synthase (i.e., the $14 \alpha$-demethylase, 14-DM)encoding gene ERG11, the overexpression of the MDR1 gene (from the major facilitator super (MFS)-family) [10, 11 , and the overexpression of the ATP-binding cassette $(\mathrm{ABC})$ transporter encoding CDR genes [12]. Among these disrupted genes, the mutation and overexpression of the 14-DM-encoding gene ERG11 have been extensively studied in relation to the drug resistance of $C$. tropicalis. The UPC2 gene encodes the zinc family transcription factor Upc2p, which exerts regulatory effects on the transcriptional level in C. albicans [13]. However, it is still unclear whether the UPC2 gene could regulate the expression of ERG11 in C. tropicalis.

In this study, the distribution and drug susceptibility of $C$. tropicalis were explored and the epidemiological characteristics of Candida isolates from a hospital setting were determined. Moreover, the relationship between ERG11 and UPC2 expression and resistance to fluconazole was investigated to provide a basis for disease diagnosis and treatment.

\section{Materials and methods}

\section{Strain sources}

The samples obtained from each clinical department of the First Affiliated Hospital of Chengdu Medical College from January 2016 to December 2019 were sent to the laboratory, and the fungi were isolated and identified based on conventional methods. For strains repeatedly obtained from the same patient at the same site, only one strain was included in the analysis.

\section{Fungal culture and preliminary identification}

The strains were inoculated onto and cultured with Sao Paulo medium (Antu Biotechnology Co., Ltd., Zhengzhou, Henan, China). The unknown fungi that were isolated were streaked on slides and subjected to Gram staining. When spores or mycelium were observed, the strain was transfected into Chromagar color development medium (Antu Biotechnology Co., Ltd.), and then it was cultured at $35^{\circ} \mathrm{C}$ for $48 \mathrm{~h}$. The fungal species were initially identified according to the different colors of the colonies.

\section{Fungal identification and antifungal susceptibility testing}

The C. tropicalis was identified by the yeast identification kit (colorimetric method) (Bio-Mérieux Co., Ltd., Lyon, France) and the minimum inhibitory concentration (MIC) of fluconazole, itraconazole, and voriconazole against C. tropicalis was determined by the yeast-like fungal susceptibility kit (microdilution method) (BioMérieux Co., Ltd.), according to the Performance Standards for Antifungal Susceptibility Testing of Yeasts (CLSI M60) recommended by the Clinical and Laboratory Standards Institute (CLSI) [14]. For fluconazole, susceptibility was defined as an $\mathrm{MIC} \leq 2 \mu \mathrm{g} / \mathrm{ml}$ and resistance $\geq 8 \mu \mathrm{g} / \mathrm{ml}$. The quality control strain for the identification and drug-sensitivity test was Candida parapsilosis (ATCC22019).

\section{Quantitative real-time PCR}

In total, 50 strains of $C$. tropicalis were randomly collected, of which, 20 strains were susceptible to fluconazole and 30 strains were resistant to fluconazole, according to the drug susceptibility test. Total RNA was extracted from exponential-phase YPD broth cultures with an RNA extraction kit (Sangon Biotechnology Co., Ltd., Shanghai, China), according to the manufacturer's instructions. Cells were collected by centrifugation at 10,000 rpm for $1 \mathrm{~min}$ and then washed in DEPCtreated $\mathrm{ddH}_{2} \mathrm{O}$. Then the cells were re-suspended in $600 \mu \mathrm{l}$ Snailase Reaction Buffer and $50 \mu \mathrm{l}$ Snailase, which was vigorously shaken. The suspension was incubated at $37{ }^{\circ} \mathrm{C}$ for $5 \mathrm{~min}$, followed by the centrifugation. The supernatant was collected, and $400 \mu \mathrm{l}$ Bufler Rlysis-Y was added. The supernatant was incubated at $65^{\circ} \mathrm{C}$ for $5 \mathrm{~min}$ and then quickly frozen at $-20{ }^{\circ} \mathrm{C}$ for $5 \mathrm{~min}$. Then $200 \mu \mathrm{l}$ Buffer YCA was added, followed by centrifugation. The supernatant was collected, and RNA was recovered by chloroform extraction, followed by ethanol precipitation, which was finally re-suspended in $50 \mu$ DEPC-treated $\mathrm{ddH}_{2} \mathrm{O}$. The cDNA template was obtained from the total RNA with the HiScript II Q RT SuperMix reverse transcription kit (Vazyme Biotechnology Co., Ltd., Nanjing, Jiangsu, China). The $2 \mu$ total RNA, $4 \mu \mathrm{l} 4 \times$ gDNA wiper Mix and $10 \mu \mathrm{l}$ RNase free $\mathrm{ddH}_{2} \mathrm{O}$ were mixed and incubated at $37{ }^{\circ} \mathrm{C}$ for $5 \mathrm{~min}$. Then $4 \mu \mathrm{l} 5 \times$ HiScript II qRT SuperMix was added, which was mixed gently. Reverse transcription was performed at $50^{\circ} \mathrm{C}$ for $15 \mathrm{~min}$ and then at $85^{\circ} \mathrm{C}$ for $5 \mathrm{~s}$ in a thermocycler. The obtained cDNAs were diluted by 10 folds with distilled water. Quantitative real-time PCR was performed with ChamQ Universal SYBR qPCR Master Mix (Vazyme Biotechnology Co., Ltd.) on a CFX96 real-time quantitative PCR instrument (BIO-RAD, California, USA). Primers were synthesized by Sangon Biotech with the following sequences: ERG11, forward $5^{\prime}$-TGCCTGGTTCTTGTTGCATTT- $3^{\prime}$ and reverse 5'-AATCGTTCAAGTCACCACCCT-3'; UPC2, forward 5'-GAGTGGAACAACAACACAACAA- $3^{\prime}$ and reverse 5'-TAAATCCCCTAAACCTGAAAGA-3'; and ACT1, forward 5'-TTTACGCTGGTTTCTCCT TGCC- $3^{\prime}$ and reverse $5^{\prime}$-GCAGCTTCCAAACCTAAA 
TCGG-3'. The reaction conditions were as follows: $95^{\circ} \mathrm{C}$ for $30 \mathrm{~s} ; 95^{\circ} \mathrm{C}$ for $10 \mathrm{~s}$, and $60{ }^{\circ} \mathrm{C}$ for $30 \mathrm{~s}$, for a total of 40 cycles. The real-time quantitative PCR quality control strain was C. tropicalis (ATCC750), and ACT1 was used as an internal reference [15].

\section{Statistical analysis}

The distribution and drug sensitivity results of fungi were analyzed with WHONET 5.6 software. The distribution of C. tropicalis was analyzed by $\chi^{2}$ test using SPSS 24.0 software. The distribution between four years was analyzed with $\chi^{2}$ test, and the Cochran $Q$ test was performed for the distribution of each year. The independent samples $t$-test was performed for the analysis of the ERG11 or UPC2 mRNA expression levels between the drug-susceptible and drug-resistant groups, and Spearman correlation analysis was also performed. $P<0.05$ was considered statistically significant.

\section{Results}

\section{Isolation of Candida}

The distribution of various types of Candida strains identified in the hospital from 2016 to 2019 was analyzed.
As shown in Fig. 1, a total of 2872 strains of Candida were identified. After the Cochran Q test, the composition ratio (i.e., the percentage of all detected C. tropicalis species) of various Candida species in each year showed statistical significance $(Q=1101.094-1904.945$; all $P<0.001$ ). For each year, $C$. albicans was the major strain, accounting for more than $60.00 \%$ of the total. $C$. tropicalis ranked third; there was no significant variation in the proportion of isolates between 2016, 2018 and 2019. Additionally, the composition ratio of each kind of Candida was compared between these four years. Our results showed statistical significance only for $C$. albicans $\left(\chi^{2}=12.620 ; P=0.006\right)$ and C. tropicalis $\left(\chi^{2}=20.410\right.$; $P<0.001$ ) (Additional file 1: Fig. S1).

\section{Sources of $C$. tropicalis samples}

The 319 strains of C. tropicalis collected from 2016 to 2019 were classified according to the sample sources, and the composition ratios were also analyzed. Our results showed that significant differences were observed in the proportion of samples from which $C$. tropicalis was detected in each year $(Q=123.949$ 194.898; $P<0.001$ ) (Fig. 2). Moreover, our results from

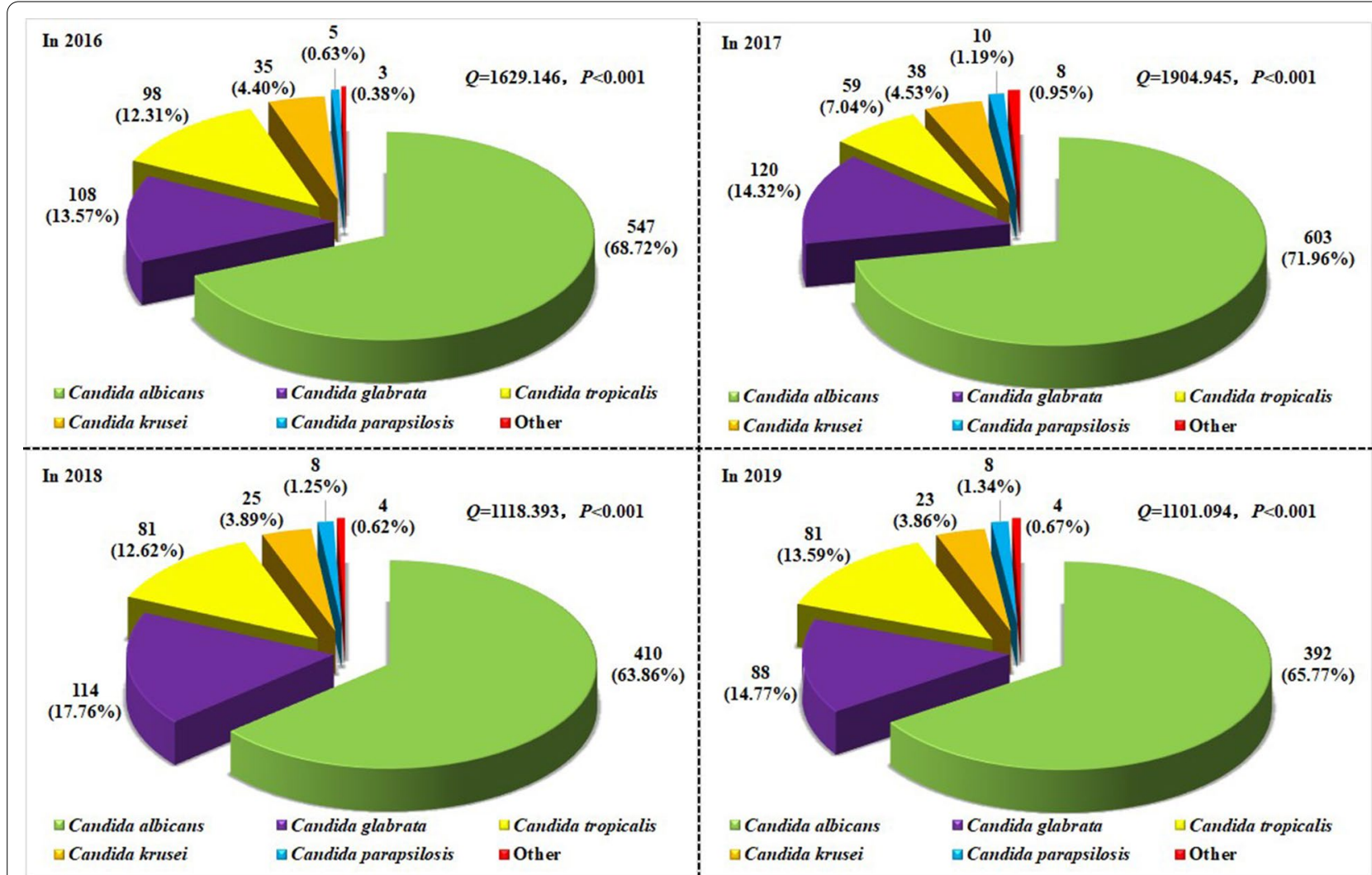

Fig. 1 Composition of Candida species detected from 2016 to 2019. The composition ratios (cases and percentage) of Candida detected in 2016, 2017, 2018, and 2019 


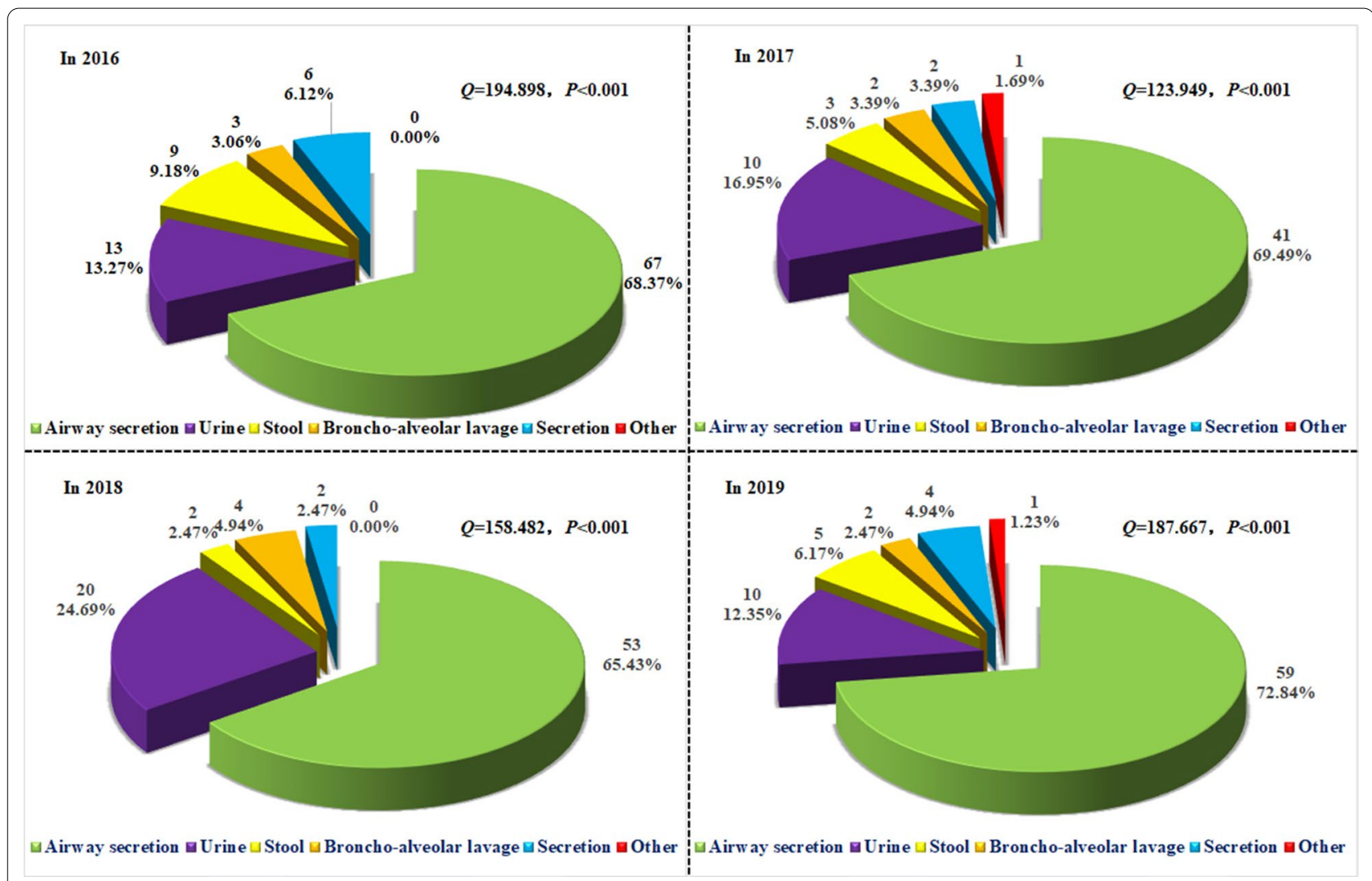

Fig. 2 Sample composition of C. tropicalis from 2016 to 2019. The sample sources (cases and percentage) of C. tropicalis detected in 2016, 2017, 2018, and 2019

the trend test showed that, except for the proportion in the stool samples $\left(\chi^{2}=3.550 ; P=0.060\right)$, the proportions in the airway secretions and urine samples increased over the years, but the proportions decreased for the other sample types year by year $($ all $P<0.050)$ (Additional file 2: Fig. S2).

\section{Department distribution of $C$. tropicalis}

The department sources of C. tropicalis from 2016 to 2019 were analyzed. Our results showed that, there were significant differences in the distribution of $C$. tropicalis in these departments from 2016 to 2019 $(Q=40.746-92.691$; all $P<0.001)$. The departments with relatively high detection rates were the Departments of Respiratory Medicine, ICU, and Geriatrics (Fig. 3). However, in comparing the C. tropicalis amount detected in each department in each of the four years, statistically significant differences were observed in the amount of $C$. tropicalis in the Department of Geriatrics $\left(X^{2}=8.623 ; P=0.035\right)$ and the ICU $\left(X^{2}=27.148 ; P<0.001\right)$ (Additional file 3: Fig. S3).

\section{Susceptibility of $C$. tropicalis to antifungal drugs}

The susceptibility to azole antifungal agents including fluconazole, itraconazole and voriconazole was analyzed in the 319 samples of C. tropicalis collected from 2016 to 2019. Our results showed that C. tropicalis had high resistance rates to fluconazole, itraconazole, and voriconazole and even exhibited cross-resistance. In 2018, the resistance rate of $C$. tropicalis to fluconazole reached $39.51 \%(32 / 81)$ and thus this year had the most resistant C. tropicalis. There were no statistically significant differences in the resistance rates of $C$. tropicalis to azole antifungal drugs over these four years $\left(\chi^{2}=1.156 ; P=0.979\right)$. However, statistical analysis found that there were significant differences in the resistance rates of $C$. tropicalis to fluconazole $\left(\chi^{2}=10.455 ; P=0.015\right)$ and voriconazole $\left(\chi^{2}=9.154 ; P=0.027\right)$ from 2016 to 2019 (Table 1$)$.

\section{Relative mRNA expression levels of ERG11 and UPC2 in C. tropicalis}

To further study the resistance-related genes of $C$. tropicalis, the mRNA expression levels of ERG11 and UPC2 were detected with quantitative real-time PCR 


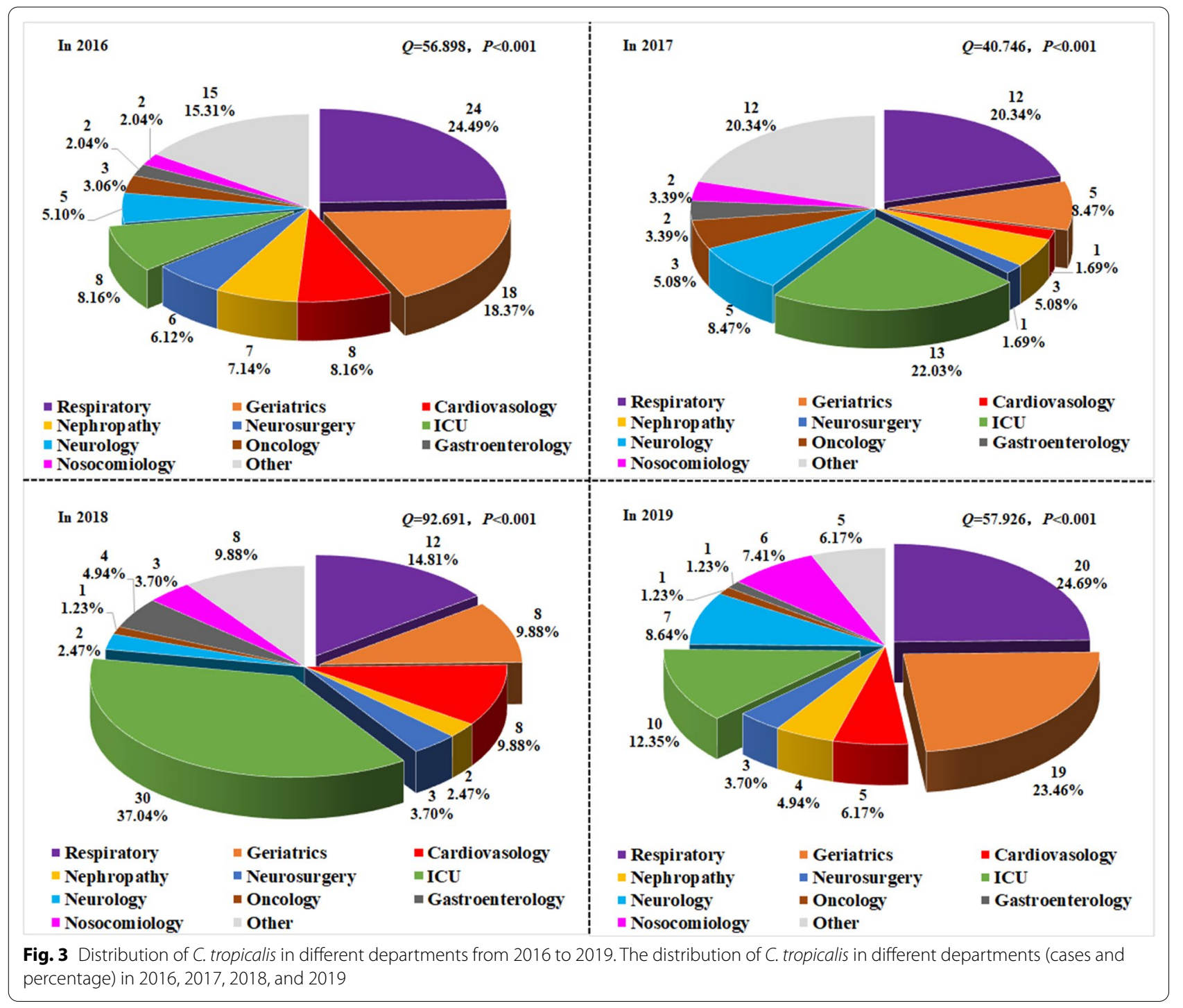

Table 1 The resistance rate of C. tropicalis to antifungal drugs from 2016 to 2019 (\%)

\begin{tabular}{lllll}
\hline Antifungal drug & $\mathbf{2 0 1 6}(\boldsymbol{n = 9 8 )}$ & $\mathbf{2 0 1 7}(\boldsymbol{n = 5 9 )}$ & $\mathbf{2 0 1 8}(\boldsymbol{n = 8 1 )}$ & $\mathbf{2 0 1 9}(\boldsymbol{n = 8 1 )}$ \\
\hline Fluconazole & $18.37(18 / 98)$ & $23.73(14 / 59)$ & $39.51(32 / 81)$ & $28.40(23 / 81)$ \\
Itraconazole & $18.37(18 / 98)$ & $28.81(17 / 59)$ & $30.86(25 / 81)$ & $23.46(19 / 81)$ \\
Voriconazole & $17.35(17 / 98)$ & $23.73(14 / 59)$ & $37.04(30 / 81)$ & $25.93(21 / 81)$ \\
\hline
\end{tabular}

$n$ is the number of strains

in 20 fluconazole-susceptible and 30 fluconazoleresistant strains extracted from 319 strains. Our results showed that the relative mRNA expression levels of ERG11 and UPC2 genes in C. tropicalis from the fluconazole-resistant group (27/30 strains fully resistant to fluconazole, itraconazole, and voriconazole) and fluconazole- susceptible group were normally distributed.
In fluconazole-resistant group, 20 strains had ERG11 overexpression and 18 strains had upregulated UPC2 expression. However, the expression level of ERG11 and UPC2 had no significant difference in the fluconazolesusceptible group. Based on the independent samples $t$-tests, the relative mRNA expression level of ERG11 in the drug-resistant group was $1.579 \pm 0.896$, while the 
relative mRNA expression of ERG11 in the susceptible group was $0.483 \pm 0.259$, and the difference was statistically significant $(t=4.511 ; P<0.001)$ (Fig. 4a). On the other hand, the relative mRNA expression level of UPC2 in the resistant group was $1.400 \pm 0.919$, while the relative mRNA expression level of UPC2 in the susceptible group was $0.448 \pm 0.272$, with a statistically significant difference $(t=3.970 ; P<0.001)$ (Fig. $4 \mathrm{~b})$. These results suggest that the resistance of $C$. tropicalis to fluconazole is related to the expression levels of ERG11 and UPC2.

\section{Correlation analysis of UPC2 and ERG11 mRNA expression} in C. tropicalis

The mRNA expression levels of resistance-related genes (i.e., ERG11 and UPC2) in C. tropicalis were detected, and the correlation between the gene expression levels was further analyzed. Our results from Spearman correlation analysis showed that there was no linear correlation between the expression levels of UPC2 and ERG11 in the susceptible group $(r=-0.074 ; P=0.757)$ (Fig. 5a). However, the UPC2 and ERG11 expression levels were positively correlated in the drug-resistance group $(r=0.571 ; P=0.001)$ (Fig. 5b).
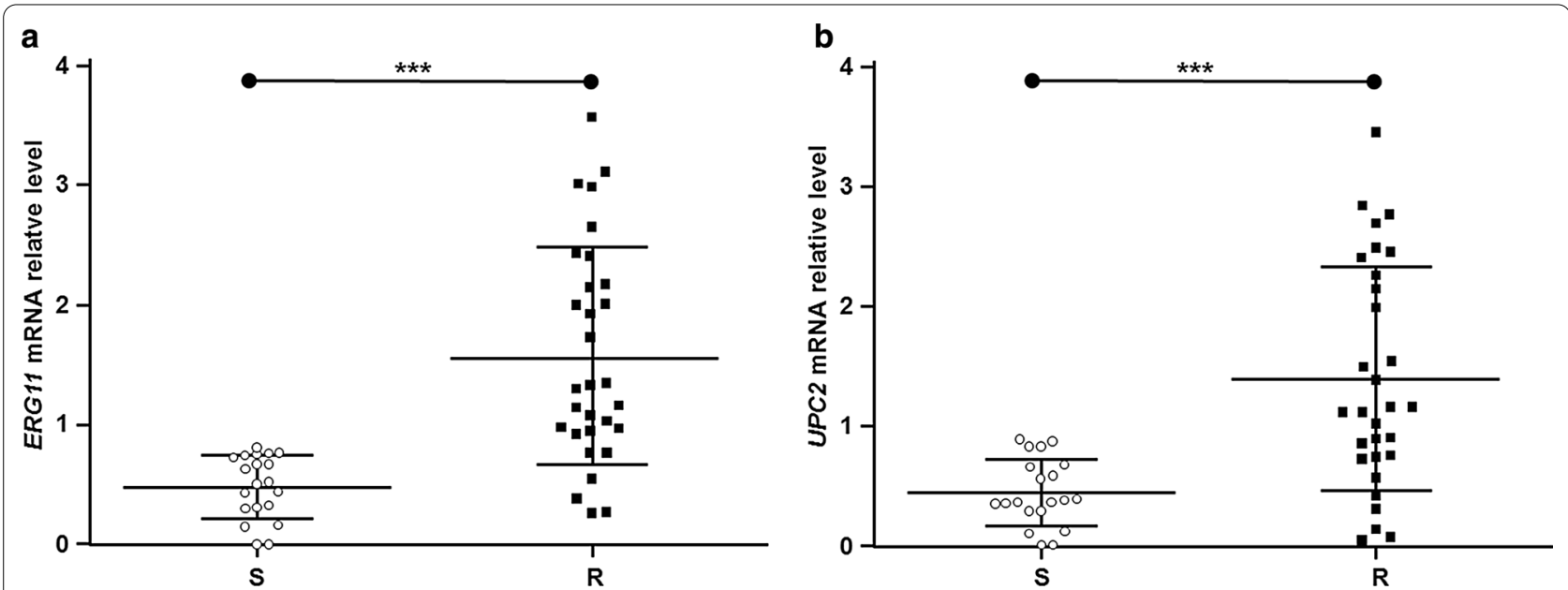

Fig. 4 ERG11 and UPC2 expression levels in C. tropicalis. (a, b) Relative mRNA expression levels of ERG11 (a) and UPC2 (b) were analyzed and compared between the susceptible group (20 strains) and the resistant group (30 strains). $P<0.001$. S, the fluconazole-susceptible group of $C$. tropicalis; and R, the fluconazole-resistant group of C. tropicalis
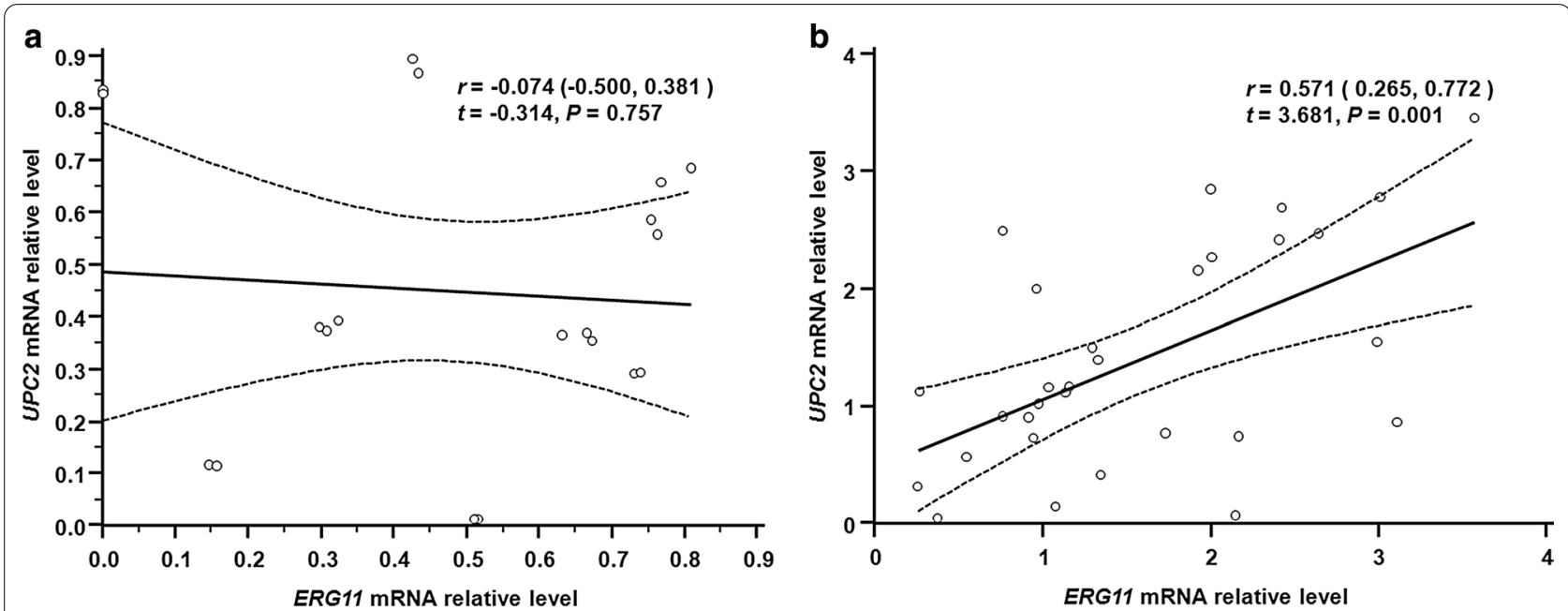

Fig. 5 Correlation analysis between UPC2 and ERG11 expression levels. $(\mathbf{a}, \mathbf{b})$ The correlation between the UPC2 and ERG11 expression levels in the fluconazole-susceptible (a) and -resistant $(\mathbf{b})$ groups of C. tropicalis. $P<0.05$ 


\section{Discussion}

C. tropicalis is a common opportunistic pathogenic fungus, and its rates of detection have been increasing over the past years. In this study, a total of 2872 strains of Candida were detected in samples collected from 2016 to 2019, of which C. albicans accounted for approximately $70.00 \%$, while $C$. tropicalis ranked third, accounting for $12.00 \%$. Moreover, the overall species composition of Candida showed statistically significant differences between the detection years. Susceptibility to antifungal agents of $C$. tropicalis in our hospital showed that the resistance rate of $C$. tropicalis to azole antifungal drugs sharply increased. In this study, based on the increasing isolation rate and drug resistance rate of C. tropicalis, the distribution and drug susceptibility of C. tropicalis were analyzed, as well as the relationship between ERG11/ UPC2 gene expression and resistance to azole antifungal drugs.

Our results showed that there was no significant difference in the overall species composition from C. tropicalis samples tested over these four years. Moreover, the main source of $C$. tropicalis was airway secretion, which might be related to that the $C$. tropicalis is one of the upper respiratory tract-colonized fungi and can be easily isolated; further, C. tropicalis has been shown to form biofilms on invasive catheters (such as tracheal tubes) [16]. Therefore, C. tropicalis with reproductive growth is often detected in airway secretions. In view of interference factors such as Candida colonization, a culture of sterile tissue or fluid should be recommended in the diagnosis of bronchopulmonary candidiasis. And then the final diagnosis should be made based on clinical manifestations and other fungal-related test results of the patients. In addition, $C$. tropicalis was detected in the mid-stream urine samples, with a detection rate of $13.00 \%-25.00 \%$, which had increased over the years. This phenomenon might be related to the increasing number of patients treated with invasive urinary tract operations and/or the increase in the number of mid-stream urine samples sent for examination. C. tropicalis has strong adhesion, penetration and destructive abilities in relation to mucous membranes, and its resistance rate to azole antifungal drugs is higher than that of C. albicans [17]. However, it is difficult to distinguish contamination, colonization or infection from urine culture. In the diagnosis of urogenital tract infection, clinicians should consider the patient's clinical manifestations, white blood cells/hyphae in the urine and the result of puncture culture.

In this study, our results showed that there were no significant differences in the resistance rate of $C$. tropicalis to azole antifungals between these four years, but the resistance rate had been increasing year by year (with the rate of resistance to fluconazole being as high as $39.51 \%$
[32/81]). The increased resistance rate of C. tropicalis to azole antifungal drugs might be related to the easy application of such drugs and the relatively mild adverse reactions [18]. In the clinic, a large number of patients with high-risk factors for invasive candidiasis were subjected to prophylaxis therapy or empirical therapy by using azole drugs to prevent the infection of Candida [19], however, long-term application of these drugs could easily lead to the emergence of azoles-resistant strains [20, 21].. Therefore, in order to slow the increase of azoleresistant C. tropicalis, the cooperation of multidisciplinary teams in hospital can make clear the diagnosis of disease, improve treatment and control antibiotic use. In addition, the diagnosis of candidiasis should be according to the high-risk factors, manifestations, etiological tests, diagnosis guidelines, etc.; the treatment of candidiasis should refer to the laboratory antifungal susceptibility test, antigen test, epidemiological characteristic, etc.; only in this way can we reasonably use azoles and avoid the prevalence of azoles-resistant Candida [22-24].

Moreover, in this study, the ERG11 and UPC2 genes of 50 strains of $C$. tropicalis were assessed, and our results showed that the relative expression level of the ERG11 gene in the drug-resistant group was higher than it was in the susceptible group, which is in line with the findings from Jiang et al. [25] regarding the high expression of ERG11 in fluconazole-resistant C. tropicalis. ERG11 overexpression could increase the amount of 14-DM in cells, which ensures ergosterol synthesis and the normal growth and reproduction of Candida, therefore leading to azole drug resistance [26, 27]. Moreover, Jiang et al. [25] cloned Y132F and S154F ERG11 mutants from C. tropicalis and introduced them into Saccharomyces cerevisiae (S. cerevisiae) and showed that the sensitivity of S. cerevisiae to azole antifungal drugs, especially fluconazole, was decreased. These results suggest that Y132F and S154F are involved in the resistance of $C$. tropicalis to fluconazole. Moreover, our results showed that the expression level of UPC2 in the resistant group was also higher than that of the susceptible group, indicating that the overexpression of UPC2 may cause $C$. tropicalis to become resistant to azole antifungal drugs, which was consistent with the findings from Jiang et al. [28]. In this study, the correlation analysis of the ERG11 and UPC2 mRNA expression levels in C. tropicalis showed that there was a linear positive correlation between the genes in the drugresistant group. These results indicated that when UPC2 was over-expressed in the fluconazole-resistant C. tropicalis, ERG11 would also be over-expressed. Therefore, the expression level of ERG11 might increase with the over-expression of UPC2. It has been shown that UPC2 has transcriptional regulation in $C$. albicans [13] and the over-expression of UPC2 in the fluconazole-resistant $C$. 
albicans can induce the over-expression of ERG11 [29, 30]. Therefore, our results suggest that the over-expression of UPC2 in the fluconazole-resistant C. tropicalis may effectively promote the over-expression of ERG11, and then increase the ergosterol synthase in cell membrane and cause the resistance to azole antifungal drugs in C. tropicalis, especially fluconazole. However, Choi et al. [31] sequenced the UPC2 gene in C. tropicalis, and their results showed that the amino acid substitutions caused by mutations in the gene appeared not only in the resistance group overexpressing ERG11, but also in the susceptible group with no ERG11 overexpression. So far, no effective missense mutation has been detected in the UPC2 of fluconazole-resistant C. tropicalis, and therefore the reason for the over-expression of UPC2 needs further study. If the expression of ERG11 and UPC2 genes can be routinely detected in clinic, the resistance of $C$. tropicalis to azole antifungal drugs can be evaluated according to the gene expression levels, which might provide more valuable guidance to the treatment of $C$. tropicalis infection. Drug resistance might also be related to multiple factors, and in a few drug-resistant $C$. tropicalis without ERG11 and UPC2 overexpression, the mechanisms underlying drug resistance might be related to efflux pumps [32] and biofilm formation [33]. To fully understand the drug resistance mechanisms of $C$. tropicalis, it is necessary to comprehensively study the impacts of the mechanism on drug sensitivity. Based on these findings, further in-depth studies are still needed to investigate the transcriptional regulatory function of Upc2p in drugresistant $C$. tropicalis and to explore how UPC2 overexpression regulates ERG11, thus leading to drug resistance to azole antifungal drugs.

\section{Conclusions}

C. tropicalis has become the most common pathogen responsible for non-C. albicans infection, and the drug resistance rate has gradually increased. It can often cause infections in patients with low immunity, basic diseases, invasive procedures, and/or long-term and large-dosage application of broad-spectrum antibiotics. Our results showed that the fluconazole resistance of $C$. tropicalis with ERG11 overexpression may be related to the regulation of the zinc family transcription factor Upc2p. Therefore, when selecting and administering azole antifungal drugs, in addition to drug sensitivity findings, clinicians should fully understand the species distribution, the formation of drug resistance, and the overexpression of ERG11 and UPC2 genes. Routine detection of ERG11 and UPC2 for high-risk patients in the clinic would contribute to early disease diagnosis and timely treatment to delay and prevent the development of resistance to $C$. tropicalis.

\section{Supplementary information}

The online version contains supplementary material available at https://doi. org/10.1186/s13756-021-00890-2.

Additional file 1: Fig. S1. Composition of various Candida species from 2016 to 2019. The composition ratios of each type of Candida detected from 2016 to 2019 were analyzed. The composition ratios of each type of Candida as a function of all types (\%) were analyzed for each year.

Additional file 2: Fig. S2. Comparison of sample composition of C. tropicalis from 2016 to 2019. The sample compositions of C. tropicalis detected from 2016 to 2019 were analyzed. The sample ratios of C. tropicalis as a function of all samples (\%) were analyzed for each year.

Additional file 3: Fig. S3. Comparison of distribution of C. tropicalis in different departments from 2016 to 2019. The distribution of C. tropicalis in different departments from 2016 to 2019 was analyzed. The composition ratios of C. tropicalis as a function of all samples (\%) in each department were analyzed for each year.

Acknowledgements

We thank Jun Luo and Lin Yin for providing C. tropicalis strains.

\section{Authors' contributions}

DW and NA conducted experiments, participated in data analysis, and drafted the initial manuscript. YY, XY and YF collected information and performed statistical analysis. JF designed the study and critically revised the manuscript. All authors read and approved the final manuscript.

Funding

This study was supported by the Chengdu Medical College program (Grant Number CYZYB20-10). The funders had no role in study design, data collection, analysis and writing of the manuscript.

Availability of data and materials

The data analyzed in this study are included in the manuscript.

\section{Ethics approval and consent to participate}

This was a retrospective study in which did not contain experiments using animals and human studies. The anonymised patient data and strains were collected and used during routine clinical practice, with patient consent. The research was approved by the Ethics Committee of the First Affiliated Hospital of Chengdu Medical College, and it was performed in accordance with the approved guidelines. Written informed consent were obtained from all participants.

\section{Consent for publication}

Not applicable.

\section{Competing interests}

The authors declare that they have no competing interests.

\section{Author details}

${ }^{1}$ Department of Laboratory Medicine, The First Affiliated Hospital of Chengdu Medical College, Chengdu 610500, Sichuan Province, China. ${ }^{2}$ Department of Laboratory Medicine, Mianyang Central Hospital, Mianyang 621000,

Sichuan Province, China.

Received: 7 April 2020 Accepted: 11 January 2021

Published online: 15 March 2021

\section{References}

1. Singh DK, Tóth R, Gácser A. Mechanisms of pathogenic Candida Species to evade the host complement attack. Front Cell Infect Microbiol. 2020;10:94

2. Zuza-Alves DL, Silva-Rocha WP, Chaves GM. An update on Candida tropicalis based on basic and clinical approaches. Front Microbiol. 2017;8:1927. 
3. Dermawan J, Ghosh S, Keating MK, Gopalakrishna KV, Mukhopadhyay S. Candida pneumonia with severe clinical course, recovery with antifungal therapy and unusual pathologic findings: a case report. Medicine. 2018;97:e9650.

4. Cuervo G, Garcia-Vidal C, Puig-Asensio M, Vena A, Meije Y, FernandezRuiz M, et al. Echinocandins compared to fluconazole for Candidemia of a urinary tract source: a propensity score analysis. Clin Infect Dis. 2017;64:1374-9.

5. Kauffman CA, Vazquez JA, Sobel JD, Gallis HA, Kinsey DS, Karchmer AW, et al. Prospective multicenter surveillance study of Funguria in hospitalized patients. The National Institute for Allergy and Infectious Diseases (NIAID) Mycoses Study Group. Clin Infect Dis. 2000;30:14-8.

6. Bassetti M, Peghin M, Carnelutti A, Righi E, Merelli M, Ansaldi F, et al. Clinical characteristics and predictors of mortality in cirrhotic patients with candidemia and intra-abdominal candidiasis: a multicenter study. Intensive Care Med. 2017;43:509-18.

7. Castanheira M, Messer SA, Rhomberg PR, Pfaller MA. Antifungal susceptibility patterns of a global collection of fungal isolates: results of the SENTRY Antifungal Surveillance Program (2013). Diagn Microbiol Infect Dis. 2016;85:200-4.

8. Fan X, Xiao M, Liao K, Kudinha T, Wang H, Zhang L, et al. Notable increasing trend in azole non-susceptible Candida tropicalis causing invasive candidiasis in China (August 2009 to July 2014):molecular epidemiology and clinical azole consumption. Front Microbiol. 2017:8:464.

9. Arastehfar A, Daneshnia F, Hafez A, Khodavaisy S, Najafzadeh MJ, Charsizadeh A, et al. Antifungal susceptibility, genotyping, resistance mechanism, and clinical profile of Candida tropicalis blood isolates. Med Mycol. 2020;58:766-73

10. Fan $X$, Xiao M, Zhang D, Huang JJ, Wang H, Hou X, et al. Molecular mechanisms of azole resistance in Candida tropicalis isolates causing invasive candidiasis in China. Clin Microbiol Infect. 2019;25:885-91.

11. Ksiezopolska E, Gabaldon T. Evolutionary emergence of drug resistance in Candida opportunistic pathogens. Genes. 2018:9:461.

12. Rocha MF, Bandeira SP, Alencar LP, Melo LM, Sales JA, Paiva M, et al. Azole resistance in Candida albicans from animals: highlights on efflux pump activity and gene overexpression. Mycoses. 2017;60:462-8.

13. Vasicek EM, Berkow EL, Flowers SA, Barker KS, Rogers PD. UPC2 is universally essential for azole antifungal resistance in Candida albicans. Eukaryot Cell. 2014;13:933-46.

14. Clinical and Laboratory Standards Institute. M60. Performance Standards for Antifungal Susceptibility Testing of Yeasts. 1st ed. Wayne: Clinical and Laboratory Standards Institute; 2017.

15. Saikat P, Shreya S, Arunaloke C, Ghosh AK. Selection and evaluation of appropriate reference genes for RTqPCR based expression analysis in Candida tropicalis following azole treatment. Sci Rep. 2020;10:1972.

16. Ladero M, Blanco M, Calderon M, Lucio L, Martin Y, Blanco M, et al. Candida tropicalis biofilm formation and expression levels of the CTRG ALS-like genes in sessile cells. Yeast. 2019;36:107-15.

17. Maria J, Marco J, Laura C, Diana G, Nancy E, Eine E, et al. Differential recognition of Candida tropicalis, Candida guilliermondii, Candida krusei, and Candida auris by human innate immune cells. Infect Drug Resist. 2019;12:783-94.

18. Kux L. Draft Guidance for Industry on Drug interaction studies-study design, data analysis, implications for dosing, and labeling recommendations; availability. Fed Reg. 2012;77:9946.
19. Pappas PG, Kauffman CA, Andes DR, Clancy CJ, Marr KA, Luis OZ, et al. Clinical practice guideline for the management of Candidiasis: 2016 update by the Infectious Diseases Society of America. Clin Infect Dis. 2016;62:e1-50.

20. Jin L, Cao Z, Wang Q, Wang Y, Wang X, Chen H, et al. MDR1 overexpression combined with ERG11 mutations induce high-level fluconazole resistance in Candida tropicalis clinical isolates. BMC Infect Dis. 2018;18:162.

21. Pfaller MA, Diekema DJ, Turnidge JD, Castanheira M, Jones RN. Twenty years of the SENTRY antifungal surveillance program: results for Candida species From 1997-2016. Open Forum Infect Dis. 2019;6(Suppl 1):79-94.

22. Scudeller L, Bassetti M, Concia E, Corrao S, Cristini F, Rosa FG, et al. MEDical wards Invasive Candidiasis ALgorithms (MEDICAL): consensus proposal for management. Eur J Intern Med. 2016;34:45-53.

23. Tissot F, Agrawal S, Pagano L, Petrikkos G, Groll AH, Skiada A, et al. ECIL-6 guidelines for the treatment of invasive candidiasis, aspergillosis and mucormycosis in leukemia and hematopoietic stem cell transplant patients. Haematologica. 2017;102:433-44.

24. Martin-Loeches I, Antonelli M, Cuenca-Estrella M, Dimopoulos G, Einav S, Waele JJ, et al. ESICM/ESCMID task force on practical management of invasive candidiasis in critically ill patients. Intensive Care Med. 2019;45:789-805

25. Jiang C, Dong D, Yu B, Cai G, Wang X, Ji Y, et al. Mechanisms of azole resistance in 52 clinical isolates of Candida tropicalis in China. J Antimicrob Chemoth. 2013;68:778-85.

26. Alizadeh F, Khodavandi A, Zalakian S. Quantitation of ergosterol content and gene expression profile of ERG11gene in fluconazole-resistant Candida albicans. Curr Med Mycol. 2017;3:13-9.

27. Feng W, Yang J, Xi Z, Qiao Z, Lv Y, Wang Y, et al. Mutations and/or overexpressions of ERG4 and ERG11 genes in clinical azoles-resistant isolates of Candida albicans. Microb Drug Resist. 2017;23:563-70.

28. Jiang C, Ni Q, Dong D, Zhang L, Li Z, Tian Y, et al. The role of UPC2 gene in azole-resistant Candida tropicalis. Mycopathologia. 2016;181:833-8.

29. Lohberger A, Coste AT, Sanglard D. Distinct roles of Candida albicans drug resistance transcription factors TAC1, MRR1, and UPC2 in virulence. Eukaryot Cell. 2014;13:127-42.

30. Sarah MP, Bassel A, Sandra W, Deken XD, Raymond M, Turcotte B. Candida albicans zinc cluster protein Upc2p confers resistance to antifungal drugs and is an activator of ergosterol biosynthetic genes. Antimicrob Agents Chemother. 2005;49:1745-52.

31. Choi MJ, Won EJ, Shin JH, Kim SH, Lee WG, Kim MN, et al. Resistance mechanisms and clinical features of fluconazole-nonsusceptible Candida tropicalis isolates compared with fluconazole-less-susceptible isolates. Antimicrob Agents Chemother. 2016;60:3653-61.

32. Shi G, Shao J, Wang T, Wu D, Wang C. Mechanism of berberine-mediated fluconazole-susceptibility enhancement in clinical fluconazole-resistant Candida tropicalis isolates. Biomed Pharmacother. 2017;93:709-12.

33. Barros PP, Rossoni RD, Ribeiro F, Junqueira JC, Jorge AO. Temporal profile of biofilm formation, gene expression and virulence analysis in Candida albicans strains. Mycopathologia. 2017;182:285-95.

\section{Publisher's Note}

Springer Nature remains neutral with regard to jurisdictional claims in published maps and institutional affiliations.

\footnotetext{
Ready to submit your research? Choose BMC and benefit from:

- fast, convenient online submission

- thorough peer review by experienced researchers in your field

- rapid publication on acceptance

- support for research data, including large and complex data types

- gold Open Access which fosters wider collaboration and increased citations

- maximum visibility for your research: over 100M website views per year
}

At BMC, research is always in progress.

Learn more biomedcentral.com/submissions 